\title{
Understanding the Mechanism of Plasmon-Driven Water Splitting: Hot Electron Injection and Near Field Enhancement Effects
}

\author{
Jiaquan Huang ${ }^{1}$, Xinyi Zhao ${ }^{2}$, Xunkun Huang ${ }^{1}$ and WanZhen Liang ${ }^{1, *}$ \\ ${ }^{1}$ State Key Laboratory of Physical Chemistry of Solid Surfaces, Fujian \\ Provincial Key Laboratory of Theoretical and Computational Chemistry, \\ and Department of Chemistry, College of Chemistry and Chemical \\ Engineering, Xiamen University, Xiamen 361005, Fujian Province, China \\ ${ }^{2}$ Xiamen Huaxia University, Ximen 361005, Fujian Province, China
}

\begin{abstract}
Utilizing plasmon-generated hot carriers to drive chemical reactions has currently become an active area of research in solar photocatalysis at the nanoscale. However, the mechanism underlying exact transfer and the generation dynamics of hot carriers, and the strategies used to further improve the quantum efficiency of the photocatalytic reaction still deserve a further look. In this work, we perform a nonadiabatic excited-state dynamics study to depict the correlation between the reaction rate of plasmon-driven water splitting (PDWS) and the sizes of gold particles, the incident light frequency and intensity, and the near-field's spatial distribution. Four model systems, $\mathrm{H}_{2} \mathrm{O}$ and $\mathrm{Au}_{20} @ \mathrm{H}_{2} \mathrm{O}$ separately interacting with the laser field and the near field generated by the Au nanoparticle (NP) with a few nanometers in size, have been investigated. Our simulated results clearly
\end{abstract}


unveil the mechanism of PDWS and hot-electron injection in a Schottky-free junction: the electrons populated on the antibonding orbitals of $\mathrm{H}_{2} \mathrm{O}$ are mandatory to drive the $\mathrm{OH}$ bond breaking and the strong orbital hybridization between $\mathrm{Au}_{20}$ and $\mathrm{H}_{2} \mathrm{O}$ creates the condition for direct electron injection. We further find that the linear dependence of the reaction rate and the field amplitude only holds at a relatively weak field and it breaks down when the second $\mathrm{OH}$ bond begins to dissociate and field-induced water fragmenting at a very intensive field, and that with the guarantee of electron injection, the water splitting rate increases with the increase of NP's size. This study will be helpful for further improving the efficiency of the photochemical reactions involving the plasmon-generated hot carriers and expanding the applications of hot carriers in varieties of chemical reactions.

\section{Introduction}

Plasmonic metal (typically $\mathrm{Au}, \mathrm{Ag}$, and $\mathrm{Cu}$ ) nanoparticles (MNPs) have extremely important applications in many fields due to their characteristic optical properties ${ }^{1-3}$ such as localized surface plasmon resonance (LSPR). When the incident light induces the collective oscillation of metal free electrons, LSPR occurs in those MNPs. ${ }^{4,5}$ However, this coherent electron oscillation can dephase quickly by nonradiative decay, ${ }^{6-9}$ generating electron-hole pairs with higher energy in non-equilibrium state (i.e., hot carriers) at the timescale of $1-100 \mathrm{fs} .{ }^{10}$ During dephasing, the collective dipole moment decreases along with the near-field interaction and scattering probabilities, with the incident energy converted into a hot carrier distribution. ${ }^{1,11}$

When molecules are adsorbed on the metal nanostructure surface, the hot electrons can be transferred to the empty states of adsorbates before thermalization and create a transient negative ion (TNI). ${ }^{12-15}$ With the response of molecular geometry, TNI moves on the excited-state potential energy surface, and therefore their reactive capability is mediated. The plasmon-induced photochemical reactions have different properties from thermal activation, and have the potential to efficiently convert solar energy into chemical energy. ${ }^{2,8,16}$ 
In general, there exist two pathways for hot electron transfer, the conventional indirect transfer and the recently proposed direct transfer. ${ }^{14,17-19}$ In indirect electron transfer process ${ }^{20}$ hot electrons are first generated in the plasmonic metal nanoparticle, then inject into the adsorbate. For direct electron transfer, ${ }^{18,21}$ in the presence of empty hybridized orbitals due to the strong metal-adsorbate coupling, chemical interface damping ${ }^{22,23}$ can induce the plasmon dephasing directly and generate nonthermalized electrons in the empty hybridized orbitals. The plasmon-induced hot carrier transfer has been utilized to foster various photochemistry processes, such as the dissociation of $\mathrm{H}_{2},{ }^{24-26} \mathrm{~N}_{2},{ }^{27}$ and $\mathrm{O}_{2},{ }^{28,29} \mathrm{CO}_{2}$ reduction, ${ }^{15,30}$ water splitting ${ }^{31-33}$ and organic transformations. ${ }^{34}$

The solar photocatalytic water splitting is a promising method for hydrogen production. This process usually take place in metal-semiconductor heterojunctions where plasmons enhance photoconversion in the semiconductor via three mechanisms, including light trapping, hot electron/hole transfer, and plasmon-induced resonance energy transfer. ${ }^{16,35}$ The solar water splitting in a Schottky-free junction has been recently found by Robatjazi et al., ${ }^{36}$ who observed large photocurrents as a result of direct injection of hot electrons from plasmonic Au NP to molecules. Many theoretical works started to explore the mechanism by investigating the effect of laser intensity and frequency on photocatalytic activity. For example, Meng's group ${ }^{31}$ simulated the $\mathrm{H}_{2} \mathrm{O}$ splitting dynamics on Au nanosphere using TDDFT/Ehrenfest dynamics scheme and found the strong correlation between laser intensity, hot electron transfer, and reaction rates. Zhang et.al. ${ }^{25}$ focused on the $\mathrm{H}_{2}$ dissociation induced by $\mathrm{Au}$ spheres and found that the dissociation rate of $\mathrm{H}_{2}$ was closely related to the molecular initial positions. In above two works, the Au particles were identified by the Jellium spheres. The Jellium model is the simplest physical model for the valence electrons, it replaces the real geometry of the metal ionic core by a featureless positively charged background in a finite volume and treats only delocalized electrons explicitly in the mean-field approximation, such as the Hartree-Fock or the local density approximations. The jellium model neglects the lattice structures of metal spheres, and hardly describes the interaction between the metal and 
adsorbates, and the effect of lattice's vibration. Furthermore, the LSPRs of MNPs are largely dependent on the MNP's shapes and sizes. It is essential to involve those effects in describing plasmon-mediated chemical reactions.

In this work, we investigate the dynamic process of water splitting driven by $\mathrm{Au}$ cluster and NPs with a few nanometers in size under femtosecond laser pulse. We explore the related mechanisms of $\mathrm{H}_{2} \mathrm{O}$ splitting and hot electron injection, and reveal the relationships between the reaction rate and the incident light intensity and frequency, and NP's sizes. The real-space real-time TDDFT/Ehrenfest dynamics schemes is adopted. Four model systems, $\mathrm{H}_{2} \mathrm{O}$ and $\mathrm{Au}_{20} @ \mathrm{H}_{2} \mathrm{O}$ interacting with the laser field and the near field generated by different sized NPs, have been investigated. The water molecule is assumed to bind with one of the vertexes of $\mathrm{Au}_{20} . \mathrm{Au}_{20}$ has a tetrahedral structure and is highly stable and chemically inert. ${ }^{37,38}$ The tetrahedral Au20 is a fragment of the face-centered cubic lattice of bulk gold with a small structural relaxation, a unique molecule with atomic packing similar to that of bulk gold. ${ }^{38}$ For $\mathrm{H}_{2} \mathrm{O}$ and $\mathrm{Au}_{20} @ \mathrm{H}_{2} \mathrm{O}$ systems, their electronic degrees of freedom were treated quantum mechanically within the real-space real-time implementation of time-dependent density functional theory (TDDFT), while their nuclei were handled classically.

Currently, it is still a great challenge to describe the excited-state dynamics of the hybrid system of molecule-MNPs with large sizes without using the jellium approximation to the MNPs. Here, we thus divide the nanosized tetrahedron into two parts: the tetrahedral cluster $\mathrm{Au}_{20}$ and the residue, where $\mathrm{Au}_{20}$ is described by TDDFT and the interaction of the residue to $\mathrm{Au}_{20} @ \mathrm{H}_{2} \mathrm{O}$ is identified by the near field to which $\mathrm{Au}_{20} @ \mathrm{H}_{2} \mathrm{O}$ is subjected. The near field is obtained by the numerical solution of Maxwell's equations using the classical computational electrodynamics finite-difference time-domain (FDTD) technique. With this regard, the interaction of metal-molecule is described at the atomic level and the strong near-field enhancement effect of NPs can also get involved. 


\section{Theoretical and computational details}

The real-space real-time TDDFT calculations reported in this work are carried out using the open-source OCTOPUS code (version 9.2). ${ }^{39-41}$ The simulation grid is localized spherically around each atom with a radius of $8.00 \AA$, and the grid point is set to be $0.20 \AA$ which is considered as the balance of simulation accuracy and time consumption. The core electrons are approximate by the Troullier-Martins pseudopotentials ${ }^{42}$ with 11, 6 and 1 valence electrons for $\mathrm{Au}, \mathrm{O}$ and $\mathrm{H}$ atoms. Therefore, the $\mathrm{Au}_{20} @ \mathrm{H}_{2} \mathrm{O}$ system has 228 valence electrons and 114 occupied orbitals, and 30 unoccupied states are included in all the calculations. The general gradient approximation exchange-correlation DFT functional Perdew-Burke-Ernzerhof $(\mathrm{PBE})^{43}$ with Average-density self-interaction correction $^{44}$ is adopted in all DFT/TDDFT calculations. The time-dependent evolution is performed using the approximated enforced time-reversal symmetry algorithm ${ }^{45}$ with a time step of 1.5 as. To obtain the optical absorption spectrum, a $\delta$ function electric field is used.

Nonadiabatic dynamics of $\mathrm{Au}_{20} @ \mathrm{H}_{2} \mathrm{O}$ is treated by TDDFT/Ehrenfest dynamics scheme $^{46}$ with temperature fluctuating at around $300 \mathrm{~K}$. The set of equations to be solved for the electron and ion dynamics of $\mathrm{Au}_{20} @ \mathrm{H}_{2} \mathrm{O}$ are

$$
\begin{aligned}
& i \frac{\partial}{\partial t} \phi_{i}(\mathbf{r}, t)=\left[\hat{h}(n(\mathbf{r}, t))+v_{\text {field }}(\mathbf{r}, t)\right], \\
& m_{\alpha} \frac{d^{2} R_{\alpha}}{d^{2} t}=F_{\alpha}(\mathbf{R}, t),
\end{aligned}
$$

where $v_{\text {field }}(\mathbf{r}, t)$ describes the time-dependent electric field acting on the system, $\left\{\phi_{i}\right\}(i=$ $1,2, \ldots, N)$ are the occupied Kohn-Sham orbitals, $\hat{h}$ is the Fock operator and $n$ is the one-electron density. $R_{\alpha}$ stands for the coordinate of the nucleus labeled $\alpha, m_{\alpha}$ for its mass, and $F_{\alpha}$ for the force exerted on it.

The laser field is assumed to polarize along the $+z$ direction with the function form: $\vec{E}_{0}(t)=\vec{E}_{\max }^{0} \exp \left[-\frac{\left(t-t_{0}\right)^{2}}{2 \tau^{2}}\right] \cos \left[\omega_{0}\left(t-t_{0}\right)\right]$, where $\left|E_{\max }^{0}\right|$ denotes the amplitude of external electric field, $\omega_{0}$ is the excitation frequency, $t_{0}=6.60 \mathrm{fs}$ is the center of Gausstype laser and $\tau=1.60 \mathrm{fs}$ is the width of laser pulse. When the water molecule is in the proximity of the nanosized $\mathrm{Au}$ tetrahedron, the field $E(r, t)$, interacted with $\mathrm{Au}_{20} @ \mathrm{H}_{2} \mathrm{O}$, 
includes the incident laser field $\vec{E}_{0}(t)$ and the scattered field $\vec{E}^{s c a}(r, t)$ generated by the truncated tetrahedron $\left(\mathrm{Au}_{20}\right.$ is cut off from this nanosized tetrahedron). This inhomogeneous near field can be expressed as $E(r, t)=E_{s}(r) E_{t}(t)$, where $E_{s}(r)$ is the spatial function that represents the non-uniform distribution of fields and $E_{t}(t)$ is the temporal function which matches the incident laser pulse. In order to describe the non-uniformity of the enhanced near-field, we need to obtain the expression of the spatial function $E_{s}(r)$. In this work, we applied fifth-order polynomial to fit the spatial function $E_{s}(r)$ via geodesic Levenberg-Marquardt (LM) algorithm, ${ }^{47,48}$ namely, $E_{s}(r) \approx a_{0}+\sum_{i=1}^{5} a_{i} r^{i}$., $r$ represent the coordinate of the space grid and $a_{i}$ are the fitting parameters.

The classical electromagnetic simulations to the Au NPs are performed using the FDTD ++ package. ${ }^{49,50}$ The Maxwell's equations are solved using Yee's algorithm. ${ }^{51,52}$ Considering that $\mathrm{Au}_{20}$ is a fragment of the face-centered cube lattice of bulk gold, or a part of the whole large-sized tetrahedron, we choose the shape of Au NP to be tetrahedral. The other reason for our choice is that we know that a weak laser field can induce an intensive near field near the apex of Au tetrahedron, even when the size of NP is small. The dielectric function of Au tetrahedron is describe by Drude-Lorentz model ${ }^{53}$ in the form of

$$
\epsilon(\omega)=\epsilon(\infty)-\frac{\omega_{D}^{2}}{\omega\left(\omega+i \gamma_{D}\right)}-\sum_{n=1}^{2} \frac{\Delta \epsilon_{L n} \omega_{L n}^{2}}{\omega\left(\omega+2 i \delta_{L n}\right)-\omega_{L n}^{2}}
$$

The parameters of dielectric function are shown in Table $1 .{ }^{54}$ The cubic simulation box with a side length of $40.00 \mathrm{~nm}$ and a grid size of $0.20 \mathrm{~nm}$ is adopted in all FDTD calculations. We use the geodesic Levenberg-Marquardt (LM) algorithm ${ }^{47,48}$ to fitting the FDTD field into a polynomial function which can be read in every TDDFT time step.We note that the current FDTD method is a classic electromagnetic model, the quantum size effect in Au NPs is ignored, which can be accounted for by the nonlocal hydrodynamic model. ${ }^{55,56}$ The neglecting of the quantum effect may bring some errors to the field enhancement. For a metal tetrahedron with the side length longer than 3 $\mathrm{nm}$, however, its quantum size effect seems not so obvious. ${ }^{57}$

The excited-state population is computed by using orbital projections. The occu- 
Table 1: The parameters of dielectric function

\begin{tabular}{cccccccc}
\hline & \multicolumn{2}{c}{ Drude } & & \multicolumn{3}{c}{ Lorentz } \\
\cline { 5 - 7 }$\epsilon_{\infty}$ & $\omega_{D}$ & $\gamma_{D}$ & & $\omega_{L n}$ & $\Delta \epsilon_{L n}$ & $\delta_{L n}$ \\
\hline \multirow{2}{*}{5.513} & 9.013 & 0.000 & 3.658 & 1.857 & 0.714 \\
& & & 2.833 & 1.004 & 0.399 \\
\hline
\end{tabular}

pation at time $t$ of the static orbital $\phi_{m}(r)$ is given by projecting all occupied timedependent orbitals onto the static unoccupied ones ${ }^{58}$

$$
p_{m}(t)=2 \sum_{j}^{o c c}\left|\left\langle\phi_{m}(0) \mid \phi_{j}(t)\right\rangle\right|^{2},
$$

and the normalization condition is $\sum_{i}^{o c c}\left|\left\langle\phi_{i}(0) \mid \phi_{j}(t)\right\rangle\right|^{2}+\sum_{m}^{u n o c c}\left|\left\langle\phi_{m}(0) \mid \phi_{j}(t)\right\rangle\right|^{2}=1$.

\section{Results and discussion}

\subsection{Electronic structure and optical properties of $\mathrm{Au}_{20} @ \mathrm{H}_{2} \mathrm{O}$}

Before investigating the dynamic processes of PDWS, we calculate the electronic and geometric structures of isolated $\mathrm{H}_{2} \mathrm{O}$ molecule and $\mathrm{Au}_{20} @ \mathrm{H}_{2} \mathrm{O}$ system. The energy gap between the highest occupied molecular orbital(HOMO) and the lowest unoccupied molecular orbital (LUMO) for the isolated $\mathrm{H}_{2} \mathrm{O}$ molecule is $8.74 \mathrm{eV}$, much larger than the energy of visible light photon, indicating that it's difficult to excite $\mathrm{H}_{2} \mathrm{O}$ to drive water splitting.

During the geometry optimization, $\mathrm{H}_{2} \mathrm{O}$ and $\mathrm{Au}_{20}$ are initially optimized separately, then they are combined to form the hybrid system of $\mathrm{Au}_{20} @ \mathrm{H}_{2} \mathrm{O}$, which is re-optimized with all $\mathrm{Au}$ atoms frozen. The optimized distance between $\mathrm{O}$ and apex of $\mathrm{Au}_{20}$ is $3.03 \AA$ as shown in the Figure 1a. The calculated density of state (DOS) shown in Figure 1 tells that the energy gap between $\mathrm{AB}$ state and HOMO of hybrid system is $2.95 \mathrm{eV}$, which is much smaller than HOMO-LUMO gap of $\mathrm{H}_{2} \mathrm{O}$, opening the possibility of excitationinduced electron transfer from $\mathrm{Au}$ cluster to the $\mathrm{AB}$ state. In addition, we plot the 
(a)

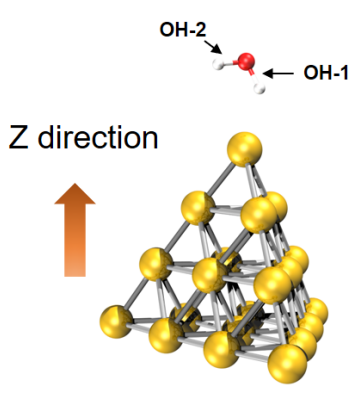

(c)

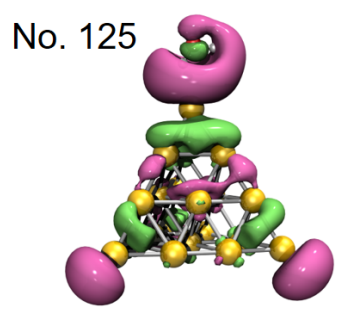

(b)

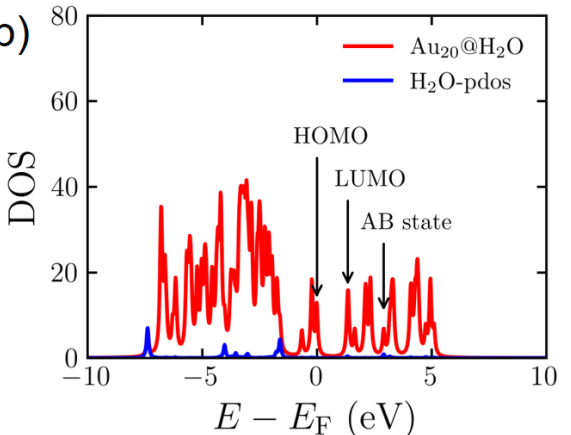

(d)

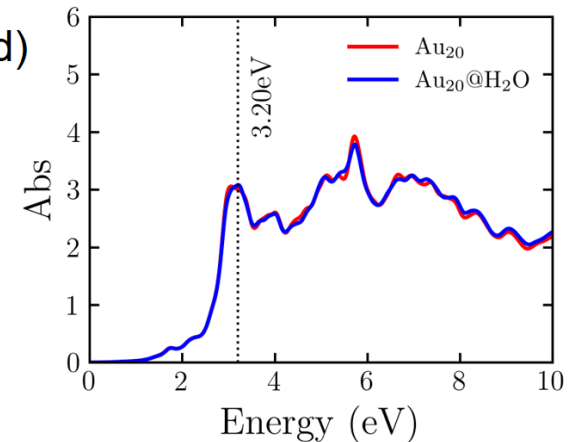

Figure 1: (a) The optimized $\mathrm{Au}_{20} @ \mathrm{H}_{2} \mathrm{O}$ geometry. (b) The DOS of $\mathrm{Au}_{20} @ \mathrm{H}_{2} \mathrm{O}(\mathrm{Fermi}$ level is set to zero) and PDOS of $\mathrm{H}_{2} \mathrm{O}$ molecule (in blue), where we mark the HOMO (No.114) and $\operatorname{LUMO}($ No.115, $1.35 \mathrm{eV})$ of the hybrid system, and the orbital with antibonding (AB) characteristics, namely, AB state (No.125, 2.95 eV). (c) The MO of No.125. (d) The absorption spectra of $\mathrm{Au}_{20} @ \mathrm{H}_{2} \mathrm{O}$ and $\mathrm{Au}_{20}$.

wavefunction of $\mathrm{AB}$ state of $\mathrm{Au}_{20} @ \mathrm{H}_{2} \mathrm{O}$ system and the optical absorption spectrum of $\mathrm{Au}_{20} @ \mathrm{H}_{2} \mathrm{O}$. For the comparison, the absorption spectrum of isolated $\mathrm{Au}_{20}$ is also plotted. The major absorption peak in low energy band of $\mathrm{Au}_{20} @ \mathrm{H}_{2} \mathrm{O}$ lies $3.20 \mathrm{eV}$, and this peak is considered to be plasmon resonance excitation. The spectral difference between $\mathrm{Au}_{20}$ and $\mathrm{Au}_{20} @ \mathrm{H}_{2} \mathrm{O}$ manifests the strong orbital coupling between the two components, can be thought to be caused by the charge transfer excitations and the interface metal/adsorbate hybrid states. ${ }^{25,29,59}$ 

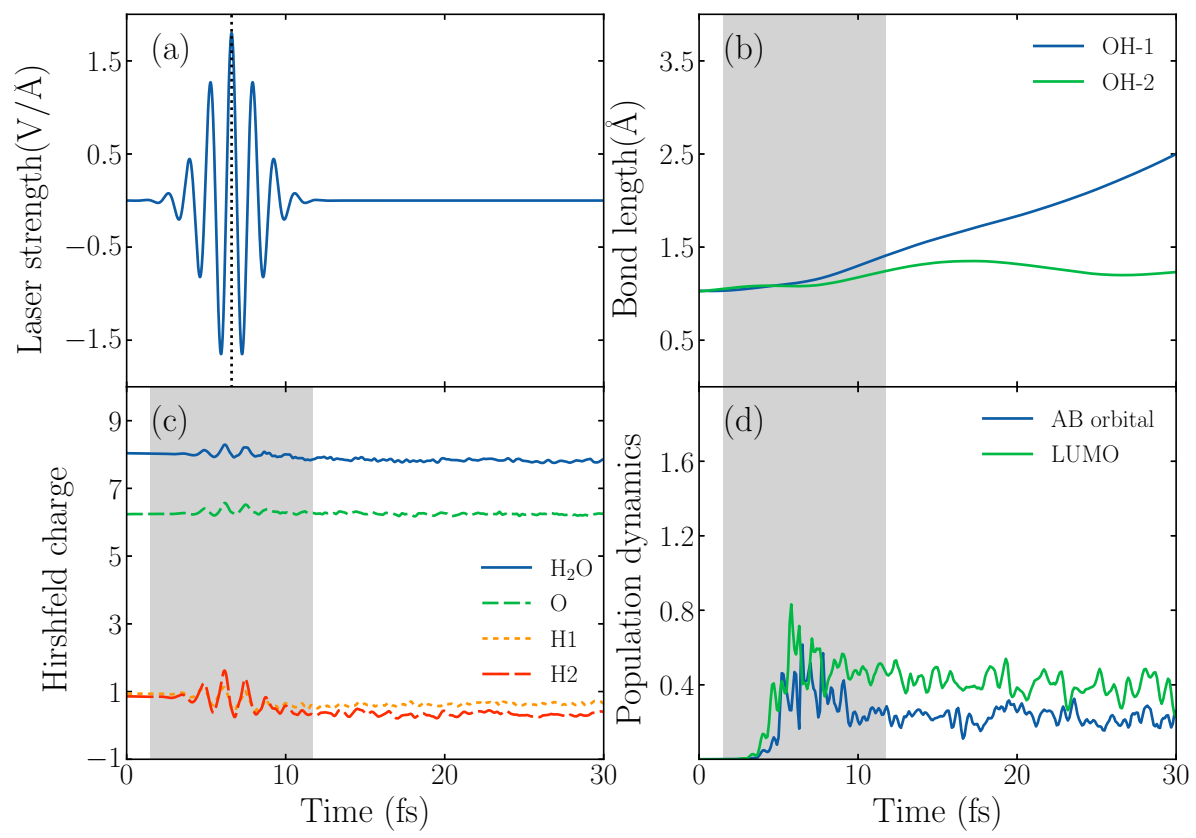

Figure 2: (a) The laser pulse with $\omega_{0}=3.07 \mathrm{eV}$ and $E_{\max }^{0}=1.80 \mathrm{~V} / \AA$ polarized in the $\mathrm{Z}$ direction. (b) Time evolution of $\mathrm{O}-\mathrm{H}$ bonds in $\mathrm{Au}_{20} @ \mathrm{H}_{2} \mathrm{O}$ system interacted with the laser field. (c) Time evolution of the Hirshfeld charge of $\mathrm{H}_{2} \mathrm{O}$ molecule. (d) The population dynamics of the LUMO (No.115) and AB state (No.125) of $\mathrm{Au}_{20} @ \mathrm{H}_{2} \mathrm{O}$. The laser illumination in the time window indicated by the gray shaded areas.

\subsection{Electronic/Nuclear dynamics of $\mathrm{Au}_{20} @ \mathrm{H}_{2} \mathrm{O}$}

In order to explore the hot electrons transfer mechanism of water splitting reaction, we show the dynamic process of $\mathrm{H}_{2} \mathrm{O}$ splitting in atomic sight by TDDFT/Ehrenfest dynamics in Figure 2, where we set $\omega_{0}=3.07 \mathrm{eV}$ and $E_{\max }^{0}=1.80 \mathrm{~V} / \AA$ (the corresponding laser intensity is $4.30 \times 10^{13} \mathrm{~W} / \mathrm{cm}^{2}$ ).

Figure $2 \mathrm{~b}$ shows the time-dependent evolution of $\mathrm{OH}$ bond length $\left(d_{\mathrm{OH}}\right)$ of the hybrid system under an intensive external laser field. The initial lengths of two $\mathrm{OH}$ bonds are $1.03 \AA$, and during the active time window of the field, one of $\mathrm{OH}$ bonds denoted by $d_{\mathrm{OH}-1}$, which is the one with $\mathrm{H}$ atom pointing to $\mathrm{Au}_{20}$ apex, increases from $1.03 \AA$ to $1.41 \AA$, and finally $d_{\mathrm{OH}-1}$ keeps increasing until it reaches to $2.49 \AA$ after $30 \mathrm{fs}$, the corresponding $\mathrm{OH}$ bond splits. However, the other $\mathrm{OH}$ bond doesn't break, $d_{\mathrm{OH}-2}$ 
increases at first and reaches the maximum value $1.35 \AA$ in $t=17.16 \mathrm{fs}$, then it decreases and oscillates until $t=30 \mathrm{fs}$. In other words, the water splitting reaction is asymmetric. The $\mathrm{OH}$ bond near the apex of $\mathrm{Au}_{20}$ is prone to breaking firstly, attributed to the initial structural arrangement. As Figure 1 shows, we set one of $\mathrm{H}$ atom of $\mathrm{H}_{2} \mathrm{O}$ to bind with $\mathrm{Au}$ atom in the apex of $\mathrm{Au}_{20}$ and the other to be away from the apex. With this structural arrangement, the symmetry of $\mathrm{H}_{2} \mathrm{O}$ breaks down. As the $\mathrm{MO}$ of No. 125 in Figure 1(c) shows, the wavefunction overlap between two $\mathrm{OH}$ bonds of $\mathrm{H}_{2} \mathrm{O}$ and $\mathrm{Au}_{20}$ will be different, indicating that their ability to accept electrons will be different. With this regard, the asymmetric dissociation appears in PDWS process.

To obtain the information of products of water splitting, we compute the Hirshfeld charge $^{60}$ of $\mathrm{H}$ and $\mathrm{O}$ atoms in real time. The amount of Hirshfeld charge may be underestimated because the electron loss when they reach the absorbing boundary. ${ }^{61}$ The results in Figure 2c tell that the Hirshfeld charge of $\mathrm{H}_{2} \mathrm{O}$ molecule oscillates with the change of external field, and the total charge number of $\mathrm{H}$ and $\mathrm{O}$ atoms fluctuates around 1 and 8 after the laser is switched off. This result indicates that $\mathrm{H}_{2} \mathrm{O}$ molecule in $\mathrm{Au}_{20} @ \mathrm{H}_{2} \mathrm{O}$ system splits into hydrogen $(\mathrm{H})$ and hydroxyl group $(\mathrm{OH})$ under this laser field.

In order to understand the reaction mechanism, we calculate the time-evolved population of $\mathrm{AB}$ state of $\mathrm{H}_{2} \mathrm{O}$ molecule and LUMO of hybrid system in Figure 2d. We observe that the photo-induced electron transfer taking place from the metal to the $\mathrm{AB}$ states of $\mathrm{H}_{2} \mathrm{O}$ molecule, indicating that the water splitting occurs when the $\mathrm{AB}$ states are populated by the electrons.

To further confirm this, we set a new system of an isolated $\mathrm{H}_{2} \mathrm{O}$ molecule interacting with the same laser field. From the result shown in Figure S2, we observe that the first $\mathrm{OH}$ bond oscillates with a period of $8.62 \mathrm{fs}$ and the maximum bond length reaches $0.94 \AA$, no signs of bond breaking. The population dynamics on HOMO and LUMO of isolated $\mathrm{H}_{2} \mathrm{O}$ molecule also tells that the rarely electron populations in $\mathrm{H}_{2} \mathrm{O}$ 's $\mathrm{AB}$ orbital, namely, this laser doesn't excite the electrons to the unoccupied orbitals of $\mathrm{H}_{2} \mathrm{O}$, so that the isolated $\mathrm{H}_{2} \mathrm{O}$ molecule doesn't split. These results suggest that the $\mathrm{OH}$ bond 
breaking in $\mathrm{Au}_{20} @ \mathrm{H}_{2} \mathrm{O}$ is attributed to the photon-induced intermolecular electronic transfer from the metal to the $\mathrm{H}_{2} \mathrm{O}$.
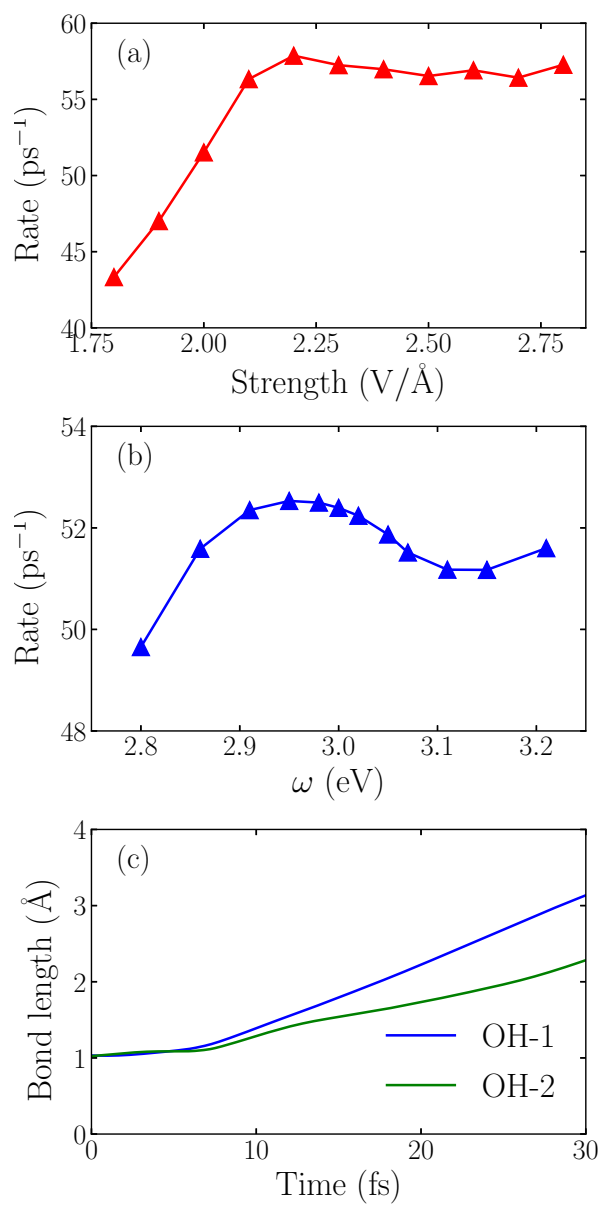

Figure 3: (a) The reaction rate varies with the intensity of the incident laser with $\omega_{0}=3.07 \mathrm{eV}$. (b) The reaction rate varies with the frequency of the incident laser with $E_{\max }^{0}=2.00 \mathrm{~V} / \AA$. (c) Time evolution of two $\mathrm{OH}$ bonds under the laser field with $E_{\max }^{0}=2.80 \mathrm{~V} / \AA$ and $\omega_{0}=3.07 \mathrm{eV}$.

Next we examine the dependence of the dissociation rate on the laser intensity and frequency. The dissociation rate of $\mathrm{H}_{2} \mathrm{O}$ molecule is defined as the inverse of time required for the first $\mathrm{OH}$ bond length to reach $2.00 \AA$. As shown in Figure 3a, initially, the splitting rate increases linearly as $E_{\text {max }}^{0}$ increases from $1.80 \mathrm{~V} / \AA$ to $2.20 \mathrm{~V} / \AA$. A maximum rate appears at $2.20 \mathrm{~V} / \AA$. When the field intensity is further increased, the rate doesn't change obviously. To get insight on this phenomenon, we plot the time- 
evolution of $\mathrm{OH}$ bond length at $E_{\max }^{0}=2.80 \mathrm{~V} / \AA$ in Figure $3 \mathrm{c}$, and find that both of two $\mathrm{OH}$ bonds are dissociated at this case. The intensive laser field can drive two $\mathrm{OH}$ bonds to break one after another though the reaction rate doesn't change obviously. As the light field increases from $1.80 \mathrm{~V} / \AA$ to $2.80 \mathrm{~V} / \AA$, there is a transition from water splitting to water fragmenting, and the dissociation rate of $\mathrm{OH}$ bond even decreases.

To unveil the possibility of water fragmentation, we perform a calculation of absorption spectra of $\mathrm{Au}_{20} @ \mathrm{H}_{2} \mathrm{O}$ with the varied laser intensity. Figure $\mathrm{S} 4$ in SI shows the calculated results. We observe that as the field intensity continuously increases, the intensities of high-energy absorption bands with excitation energies $\geq 2.9 \mathrm{eV}$ decrease and their peak locations blue-shift while the intensities of low-energy absorption bands continuingly increase. These phenomena manifest that the intensive laser fields couple with the system $\mathrm{Au}_{20} @ \mathrm{H}_{2} \mathrm{O}$ nonlinearly. ${ }^{64}$ The decrease of the high-energy absorption and the integrated energy shift are attributed to the absorption saturation and the field dressing during the excitation. The appearing and continuing increasing intensity of low-energy bands confirms that the multiphoton absorption occurs. This nonlinear coupling between the system and laser field definitely affects the dynamics of water splitting and may lead to the water fragmentation.

Figure $3 \mathrm{~b}$ shows that the splitting rate varies with the laser frequency. The maximum rate appears at $\omega_{0}=2.95 \mathrm{eV}$, not at $3.20 \mathrm{eV}$, indicating that more hot electrons are transferred when the frequency of the incident field matches the energy gap between Fermi-level and $\mathrm{AB}$ orbital, namely, the hot electrons injection mechanism in water splitting reaction follows the direct one. ${ }^{11,25}$

\subsection{Electronic/Nuclear dynamics of nanosized tetrahedron@ $\mathrm{H}_{2} \mathrm{O}$}

To simulate the plasmon-induced water splitting reaction with Au particle at the nanometer scale with mixed TDDFT/FDTD method, we firstly calculate the time-dependent spatial inhomogeneous scattering field generated by the truncated Au tetrahedron via FDTD ++ package, then describe the time evolution trajectory dynamics of $\mathrm{Au}_{20} @ \mathrm{H}_{2} \mathrm{O}$ system under the intensive near field by TDDFT/Ehrenfest scheme. With this treatmen- 


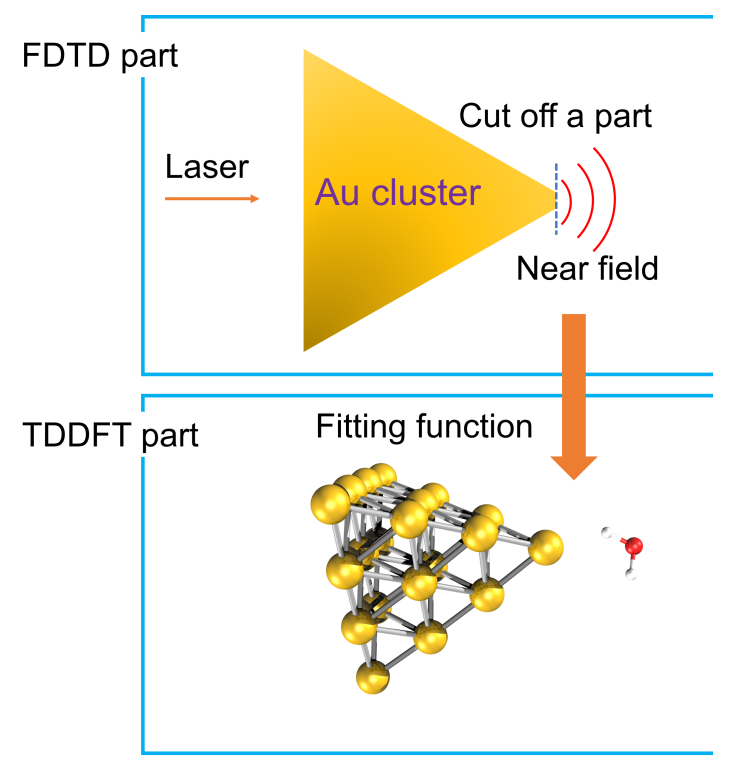

Figure 4: The schematic diagram showing the mixed FDTD/TDDFT scheme for the PDWS reaction with the large-sized NP.

$\mathrm{t}$, the hot electron injection is assumed to always appear around the interface between the metal cluster and the adsorbate, and the different sized NP provides the near field with different intensity to which $\mathrm{Au}_{20} @ \mathrm{H}_{2} \mathrm{O}$ is subjected, as Figure 4 shows.

Figure 5 shows the time evolution of the $\mathrm{OH}$ bond length in $\mathrm{Au}_{20} @ \mathrm{H}_{2} \mathrm{O}$ under the intensive near field which generated by the truncated $\mathrm{Au}$ tetrahedrons with different sizes. Here the laser field with $E_{\max }^{0}=0.50 \mathrm{~V} / \AA$ and $\omega_{0}=3.07 \mathrm{eV}$ is set. We observe that one of $\mathrm{OH}$ bonds breaks at $25.12 \mathrm{fs}$ under the field enhancement by the truncated $\mathrm{Au}$ tetrahedron with $\mathrm{L}=2.88 \mathrm{~nm}$. In this case, the disassociation reaction takes place at $E_{\max }^{0}<1.80 \mathrm{~V} / \AA$. Furthermore, we observe that the larger is the NP's size, the faster is the disassociation reaction. For example, at $\mathrm{L}=4.32 \mathrm{~nm}$, the two $\mathrm{OH}$ bonds's dissociation times are $21.47 \mathrm{fs}$ and $27.80 \mathrm{fs}$ while at $\mathrm{L}=5.76 \mathrm{~nm}$, they are $21.03 \mathrm{fs}$ and $25.88 \mathrm{fs}$, respectively. For comparison, we also calculate the splitting dynamics of $\mathrm{H}_{2} \mathrm{O}$ molecule which interacts with the near field generated by the whole Au tetrahedron of $\mathrm{L}=2.88 \mathrm{~nm}$ obtained by FDTD. In this case, no electron is injected to $\mathrm{H}_{2} \mathrm{O}$, and only the field enhancement effect of $\mathrm{Au}$ NPs gets involved. As Figure 5 shows, OH 


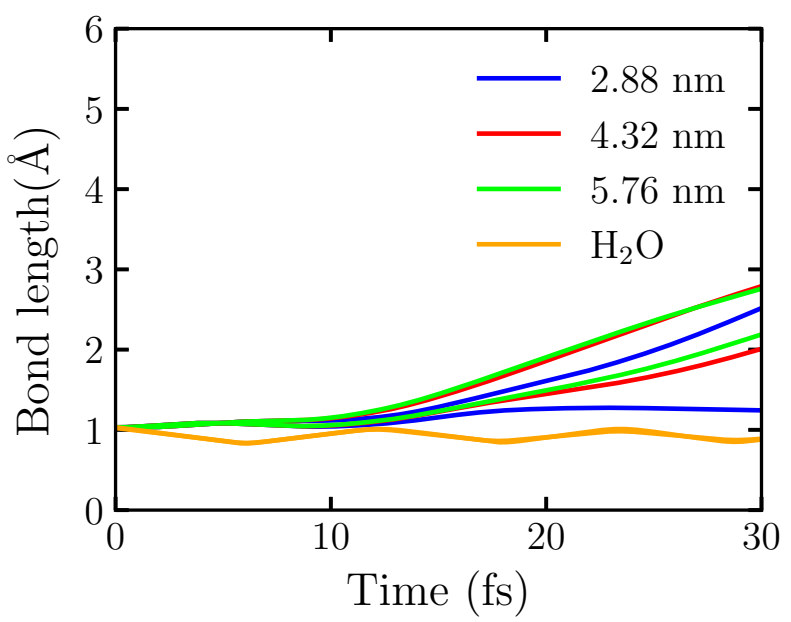

Figure 5: Time evolution of $\mathrm{OH}$ bonds in $\mathrm{Au}_{20} @ \mathrm{H}_{2} \mathrm{O}$ interacted with the intensive near field generated by the truncated Au tetrahedron with different side length of $2.88 \mathrm{~nm}$, $4.32 \mathrm{~nm}$, and $5.76 \mathrm{~nm}$, respectively. The orange line represents the time evolution of $\mathrm{OH}$ bonds in the isolated $\mathrm{H}_{2} \mathrm{O}$ interacted with the near field generated by the whole $\mathrm{Au}$ tetrahedron of $\mathrm{L}=2.88 \mathrm{~nm}$ (without the truncation of $\mathrm{Au}_{20}$ ).

bond oscillates at a certain frequency, and there is no sign of splitting. These numerical simulations suggest that the near field can enhance the reaction rate of water splitting and one can adjust the size of metal NP to control the plasmon-assisted photochemistry via field enhancement effect. However, without the injection of hot electrons, the water splitting reaction will not take place, which highlights the decisive factor to drive the water splitting.

To show the field enhancement effect, we calculate the absorption spectra and near fields of the truncated $\mathrm{Au}$ tetrahedrons with different side lengths of $2.88 \mathrm{~nm}, 4.32 \mathrm{~nm}$, and $5.76 \mathrm{~nm}$ in Figure S5 and Figure S6. The major low-energy absorbtion bands locate at $1.92 \sim 2.16 \mathrm{eV}$, deviated largely from the major low-energy absorption peak of $\mathrm{Au}_{20}$, manifesting the quantum size effect of optical responses of NPs with a few nanometers in size. The larger is the NP's size, the stronger is the absorption. And then, we calculate the strong scattered field generated by the truncated $\mathrm{Au}$ tetrahedrons. The incident laser is set to match the one used in TDDFT calculation. Figure S6 clearly shows the 
strong field enhancement effect near the 'tip' of tetrahedron, and the 'hot spots' are formed close to the $\mathrm{Au}$ particles. In addition, it is clear that the field enhancement rate increases with the size of NPs.

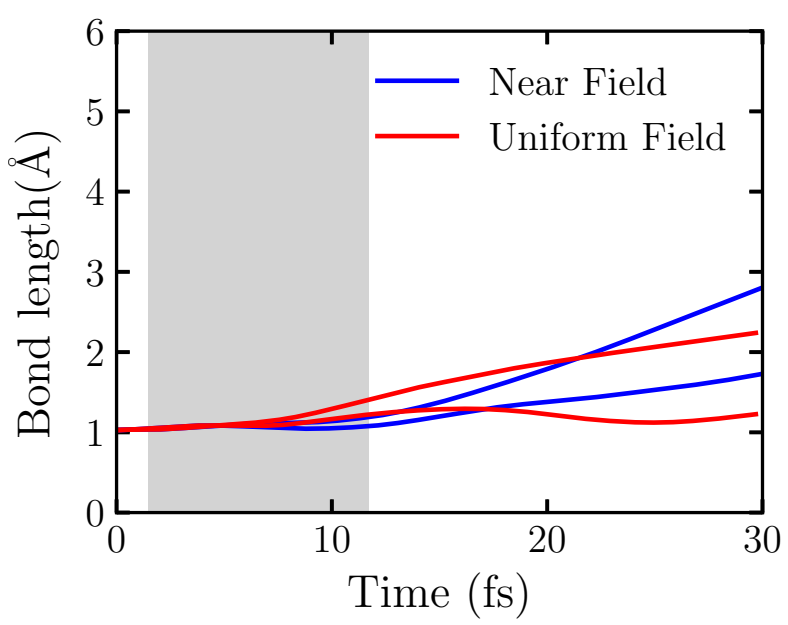

Figure 6: Time evolution of $\mathrm{OH}$ bonds of $\mathrm{Au}_{20} @ \mathrm{H}_{2} \mathrm{O}$ interacting with the real near field and the uniform electric field, respectively. Here the laser field with $E_{\max }^{0}=0.65 \mathrm{~V} / \AA$ and $\omega_{0}=3.07 \mathrm{eV}$ is applied.

The near field decays rapidly with the surface separation as Figure S6 shows. To have a clear picture on the effect of the near field's inhomogeneity on the water splitting, we perform the TDDFT/Ehrenfest dynamics of $\mathrm{Au}_{20} @ \mathrm{H}_{2} \mathrm{O}$ interacted with the real near field generated by the truncated Au tetrahedron with $\mathrm{L}=2.88 \mathrm{~nm}$ and the uniform electric field taken from the center of mass of $\mathrm{Au}_{20} @ \mathrm{H}_{2} \mathrm{O}$ generated by the same NP, respectively. In this case, a very weak laser field with $\omega_{0}=3.07 \mathrm{eV}$ and $E_{\max }^{0}=0.65 \mathrm{~V} / \AA$ is applied. As Figure S7 shows, the duration of scattered field at the center of mass of $\mathrm{Au}_{20} @ \mathrm{H}_{2} \mathrm{O}$ is much longer than the incident field, the scattered field's amplitude is magnified about three-fold so that the $\mathrm{OH}$ bond disassociates even with a weak laser field. Figure 6 displays the evolution of $\mathrm{OH}$ bonds in $\mathrm{Au}_{20} @ \mathrm{H}_{2} \mathrm{O}$ which is interacting with the two kinds of fields, respectively. In the laser activity window, in the case with a uniform field, the change of $\mathrm{OH}$ bond length shows an "upward arc", while in the real near field case, it shows as a "downward arc". The near field generated from LSPR is a very 
short-range electromagnetic field with a strong intensity gradient which may generate gradient force to affect the molecule. ${ }^{62,63}$

To explain why the field's spatial distribution can affect the evolution of $\mathrm{OH}$ bonds with the time, we compare the forces acting on $\mathrm{H}_{2} \mathrm{O}$ molecule in Figure $\mathrm{S} 8$ and Figure S9. During the laser activity window ( from $6.60 \mathrm{fs}$ to $6.84 \mathrm{fs}$ ), the results with the uniform field and the near field are quite dissimilar. In the uniform field, the force vectors in the $X Z$ plane act on the $\mathrm{H}_{2} \mathrm{O}$ molecule are affected by the field and electron injection, and its direction and magnitude change rapidly. While in the near field, the force vectors in the $X Z$ plane always point to the 'tips' of $\mathrm{Au}_{20}$, especially the force acting on $\mathrm{O}$ atom. It is known that this direction is also the negative gradient direction of the field. We thus suggest that the inhomogeneous near field affects the water splitting reaction through the gradient force and causes a different reaction during the field active window.

\section{Concluding Remarks}

We have presented a theoretical study on the PDWS via TDDFT/Ehrenfest nonadiabatic dynamics. By comparing the calculated results of four model systems: $\mathrm{H}_{2} \mathrm{O}$ and $\mathrm{Au}_{20} @ \mathrm{H}_{2} \mathrm{O}$ separately interacting with the laser field and the near fields, we clearly reveal the microscopic mechanism of PDWS and the interface electron transfer, and the correlation of the reaction rate with the laser field frequency and intensity, the NP's sizes, and the field's spatial distribution. A multiscale scheme has been applied to describe PDWS dynamics. For $\mathrm{H}_{2} \mathrm{O}$ and $\mathrm{Au}_{20} \mathrm{QH}_{2} \mathrm{O}$ systems, its electronic degrees of freedom were treated quantum mechanically within the real-space real-time implementation of TDDFT, while their ions were handled classically. For the system with larger sized NP, we divided this NP into $\mathrm{Au}_{20}+$ residue, where $\mathrm{Au}_{20}$ was described by TDDFT and the truncated NP was described by FDTD.

The main conclusions are summarized as follows:

(1) The electrons populated on the $\mathrm{AB}$ orbitals of $\mathrm{H}_{2} \mathrm{O}$ are mandatory to drive the $\mathrm{OH}$ bond breaking. The strong orbital hybridization between $\mathrm{Au}_{20}$ and $\mathrm{H}_{2} \mathrm{O}$ creates the 
condition for photo-induced direct electron injection.

(2) The dynamic results under different laser intensity unveil that the linear dependence of reaction rate of PDWS and the incident field amplitude holds only at a relatively weak field, however the linear correlation breaks down by the participation of other reactions such as water fragmenting in the intensive field regime. The splitting rate varies with the laser frequency, and the maximum rate appears when the laser frequency matches the energy gap between the metal Fermi-level and AB orbital.

(3) To describe the effect of field enhancement induced by large-sized Au NPs, the mixed FDTD/TDDFT method is used. By dividing the large sized NP into $\mathrm{Au}_{20}+$ Residue, the requirement of near-field enhancement and hot-electron injection in the water splitting reaction can be simultaneously satisfied. Setting the water near the apex of large-sized tetrahedron, $\mathrm{OH}$ bonds cab be disassociated by a very weak laser field.

(4)The intensive electric field can make two $\mathrm{OH}$ bonds in $\mathrm{Au}_{20} @ \mathrm{H}_{2} \mathrm{O}$ system break successively, attributed to the nonlinear coupling between the system and the applied field. When the intensity of the incident field reaches a certain degree, the field couples with the system nonlinearly, opening the possibility of multiphoton absorption. These hot electron excited by multiphoton absorption can give important contributions in $\mathrm{H}_{2} \mathrm{O}$ splitting reaction.

(5) The influence of the field's spatial distribution on water splitting is significant. The gradient force caused by the strong intensity gradient of the near field makes the distinction of force vectors in $X Z$ plane compared to those in the uniform field case, leading to the different $\mathrm{OH}$ bond evolution dynamics.

This work is useful for understanding the hot electron induced reactions at ambient conditions by plasmonic excitations and can provide a reference for the development of related mixed quantum-classical method in describe the large-sized plasmonic system. 


\section{Support Information Available}

The materials in SI include the DOS of isolated $\mathrm{H}_{2} \mathrm{O}$ molecule, the evolution of $\mathrm{OH}$ bond when an isolated $\mathrm{H}_{2} \mathrm{O}$ molecule interacts with the laser field, the time evolution of $\mathrm{OH}$ bonds in $\mathrm{Au}_{20} @ \mathrm{H}_{2} \mathrm{O}$ system under the laser field with $E_{\max }=1.60 \mathrm{~V} / \AA$ and $E_{\max }=1.70 \mathrm{~V} / \AA$ ), the absorption spectra of $\mathrm{Au}_{20} @ \mathrm{H}_{2} \mathrm{O}$ vary with the intensity of incident field, the absorption spectra of Au tetrahedrons with $\mathrm{L}=2.88 \mathrm{~nm}, 4.32 \mathrm{~nm}$, and $5.76 \mathrm{~nm}$ calculated by FDTD, the contour plot of near fields, the time evolution of the forces acted on $\mathrm{H}_{2} \mathrm{O}$ molecule in the $X Z$ plane under a uniform field and the real near field, respectively.

\section{Acknowledgements}

The financial support from the National Natural Science Foundation of China with grant No.21833006 is appreciated.

\section{AUTHOR INFORMATION}

\section{Corresponding Author}

*E-mail: liangwz@xmu.edu.cn

\section{References}

[1] M. Bernardi, J. Mustafa, J. B. Neaton and S. G. Louie, Nat. Commun., 2015, 6, 1-9.

[2] V. Giannini, A. I. Fernández-Domínguez, S. C. Heck and S. A. Maier, Chem. Rev., 2011, 111, 3888-3912.

[3] G. V. Hartland, Chem. Rev., 2011, 111, 3858-3887. 
[4] N. J. Halas, S. Lal, W.-S. Chang, S. Link and P. Nordlander, Chem. Rev., 2011, 111, 3913-3961.

[5] K. A. Willets and R. P. Van Duyne, Annu. Rev. Phys. Chem., 2007, 58, 267-297.

[6] L. D. Landau, Zh Eksp Teor Fiz, 1946, 10, 25.

[7] D. Ryutov, Plasma Phys. Control. Fusion, 1999, 41, A1.

[8] M. L. Brongersma, N. J. Halas and P. Nordlander, Nat. Nanotechnol., 2015, 10, $25-34$.

[9] X. Li, D. Xiao and Z. Zhang, New J. Phys., 2013, 15, 023011.

[10] C. Voisin, N. Del Fatti, D. Christofilos and F. Vallée, J. Phys. Chem. B, 2001, 105, $2264-2280$.

[11] H. Tang, C.-J. Chen, Z. Huang, J. Bright, G. Meng, R.-S. Liu and N. Wu, J. Chem. Phys., 2020, 152, 220901.

[12] J. S. DuChene, B. C. Sweeny, A. C. Johnston-Peck, D. Su, E. A. Stach and W. D. Wei, Angew. Chem. - Int. Ed., 2014, 53, 7887-7891.

[13] S.-F. Hung, F.-X. Xiao, Y.-Y. Hsu, N.-T. Suen, H.-B. Yang, H. M. Chen and B. Liu, Adv. Energy Mater., 2016, 6, 1501339.

[14] M. J. Kale, T. Avanesian, H. Xin, J. Yan and P. Christopher, Nano Lett., 2014, 14, $5405-5412$.

[15] S. Yu, A. J. Wilson, J. Heo and P. K. Jain, Nano Lett., 2018, 18, 2189-2194.

[16] S. K. Cushing and N. Wu, J. Phys. Chem. Lett., 2016, 7, 666-675.

[17] Q. Wei, S. Wu and Y. Sun, Adv. Mater., 2018, 30, 1802082.

[18] C. Boerigter, U. Aslam and S. Linic, Acs Nano, 2016, 10, 6108-6115.

[19] C. Boerigter, R. Campana, M. Morabito and S. Linic, Nat. Commun., 2016, 7, 1-9. 
[20] Y. Tian and T. Tatsuma, J. Am. Chem. Soc., 2005, 127, 7632-7637.

[21] Q. Xiao, S. Sarina, E. R. Waclawik, J. Jia, J. Chang, J. D. Riches, H. Wu, Z. Zheng and H. Zhu, Acs Catal., 2016, 6, 1744-1753.

[22] H. Hövel, S. Fritz, A. Hilger, U. Kreibig and M. Vollmer, Phys. Rev. B, 1993, 48, 18178.

[23] B. Foerster, A. Joplin, K. Kaefer, S. Celiksoy, S. Link and C. Sönnichsen, Acs Nano, 2017, 11, 2886-2893.

[24] L. Yan, Z. Ding, P. Song, F. Wang and S. Meng, Appl. Phys. Lett., 2015, 107, 083102.

[25] Y. Zhang, T. Nelson, S. Tretiak, H. Guo and G. C. Schatz, Acs Nano, 2018, 12, $8415-8422$.

[26] Q. Wu, L. Zhou, G. C. Schatz, Y. Zhang and H. Guo, J. Am. Chem. Soc., 2020, 142, 13090-13101.

[27] O. A. Hull, D. B. Lingerfelt, X. Li and C. M. Aikens, J. Phys. Chem. C, 2020, 124, 20834-20845.

[28] P. Christopher, H. Xin, A. Marimuthu and S. Linic, Nat. Mater., 2012, 11, 1044 1050 .

[29] B. Seemala, A. J. Therrien, M. Lou, K. Li, J. P. Finzel, J. Qi, P. Nordlander and P. Christopher, ACS Energy Lett., 2019, 4, 1803-1809.

[30] H. Robatjazi, H. Zhao, D. F. Swearer, N. J. Hogan, L. Zhou, A. Alabastri, M. J. McClain, P. Nordlander and N. J. Halas, Nat. Commun., 2017, 8, 1-10.

[31] L. Yan, F. Wang and S. Meng, Acs Nano, 2016, 10, 5452-5458.

[32] K. Ueno, T. Oshikiri and H. Misawa, Chemphyschem, 2016, 17, 199-215.

[33] L. Yan, J. Xu, F. Wang and S. Meng, J. Phys. Chem. Lett., 2018, 9, 63-69. 
[34] H. Kisch, Angew. Chem. - Int. Ed., 2013, 52, 812-847.

[35] Z. Zheng, W. Xie, B. Huang and Y. Dai, Chem. Eur. J., 2018, 24, 18322-18333.

[36] H. Robatjazi, S. M. Bahauddin, C. Doiron and I. Thomann, Nano Lett., 2015, 15, 6155-6161.

[37] R. W. Burgess and V. J. Keast, J. Phys. Chem. C, 2014, 118, 3194-3201.

[38] J. Li, X. Li, H.-J. Zhai and L.-S. Wang, Science, 2003, 299, 864-867.

[39] A. Castro, H. Appel, M. Oliveira, C. A. Rozzi, X. Andrade, F. Lorenzen, M. A. Marques, E. Gross and A. Rubio, Phys. Status Solidi B, 2006, 243, 2465-2488.

[40] X. Andrade, D. Strubbe, U. De Giovannini, A. H. Larsen, M. J. Oliveira, J. AlberdiRodriguez, A. Varas, I. Theophilou, N. Helbig, M. J. Verstraete et al., Phys. Chem. Chem. Phys., 2015, 17, 31371-31396.

[41] N. Tancogne-Dejean, M. J. Oliveira, X. Andrade, H. Appel, C. H. Borca, G. Le Breton, F. Buchholz, A. Castro, S. Corni, A. A. Correa et al., J. Chem. Phys., 2020, 152, 124119.

[42] N. Troullier and J. L. Martins, Phys. Rev. B, 1991, 43, 1993.

[43] J. P. Perdew, K. Burke and M. Ernzerhof, Phys. Rev. Lett., 1996, 77, 3865.

[44] C. Legrand, E. Suraud and P. Reinhard, J. Phys. B: At. Mol. Opt. Phys., 2002, 35, 1115.

[45] A. Castro, M. A. Marques and A. Rubio, J. Chem. Phys., 2004, 121, 3425-3433.

[46] J. L. Alonso, X. Andrade, P. Echenique, F. Falceto, D. Prada-Gracia and A. Rubio, Phys. Rev. Lett., 2008, 101, 096403.

[47] M. K. Transtrum, B. B. Machta and J. P. Sethna, Phys. Rev. Lett., 2010, 104, 060201. 
[48] M. K. Transtrum, B. B. Machta and J. P. Sethna, Phys. Rev. E, 2011, 83, 036701.

[49] A. L. Atkinson, J. M. McMahon and G. C. Schatz, in SelfOrganization of Molecular Systems, Springer, 2009, pp. 11-32.

[50] J. M. McMahon, Y. Wang, L. J. Sherry, R. P. Van Duyne, L. D. Marks, S. K. Gray and G. C. Schatz, J. Phys. Chem. C, 2009, 113, 2731-2735.

[51] K. Yee, IEEE Trans. Antennas Propag., 1966, 14, 302-307.

[52] E. Miller, J. Electromagnet. Wave., 1994, 8, 1125-1172.

[53] P. Drude, Ann. Phys-berlin., 1900, 308, 369-402.

[54] J. M. McMahon, G. C. Schatz and S. K. Gray, Phys. Chem. Chem. Phys., 2013, 15, 5415-5423.

[55] J. M. McMahon, S. K. Gray and G. C. Schatz, Nano Lett., 2010, 10, 3473-3481.

[56] C. Ciracì, R. Hill, J. Mock, Y. Urzhumov, A. Fernández-Domínguez, S. Maier, J. Pendry, A. Chilkoti and D. Smith, Science, 2012, 337, 1072-1074.

[57] P. Zhang, J. Jing and W. Z. Liang, J. Phys. Chem. C, 2018, 122, 10545.

[58] J. Krumland, A. M. Valencia, S. Pittalis, C. A. Rozzi and C. Cocchi, J. Chem. Phys., 2020, 153, 054106.

[59] R. L. Gieseking, M. A. Ratner and G. C. Schatz, in Frontiers of Plasmon Enhanced Spectroscopy Volume 1, ACS Publications, 2016, pp. 1-22.

[60] F. L. Hirshfeld, Theor. Chim. Acta, 1977, 44, 129-138.

[61] A. Castro, E. Räsänen, A. Rubio and E. Gross, Europhys. Lett., 2009, 87, 53001.

[62] C. Zhan, G. Wang, J. Yi, J.-Y. Wei, Z.-H. Li, Z.-B. Chen, J. Shi, Y. Yang, W. Hong and Z.-Q. Tian, Matter, 2020, 3, 1350-1360.

[63] T. Iwasa and K. Nobusada, Phys. Rev. A, 2010, 82, 043411. 
[64] J. Sun, Z. Ding, Y. Yu and W. Liang, Phys. Chem. Chem. Phys., 2021, 23, 1599416004

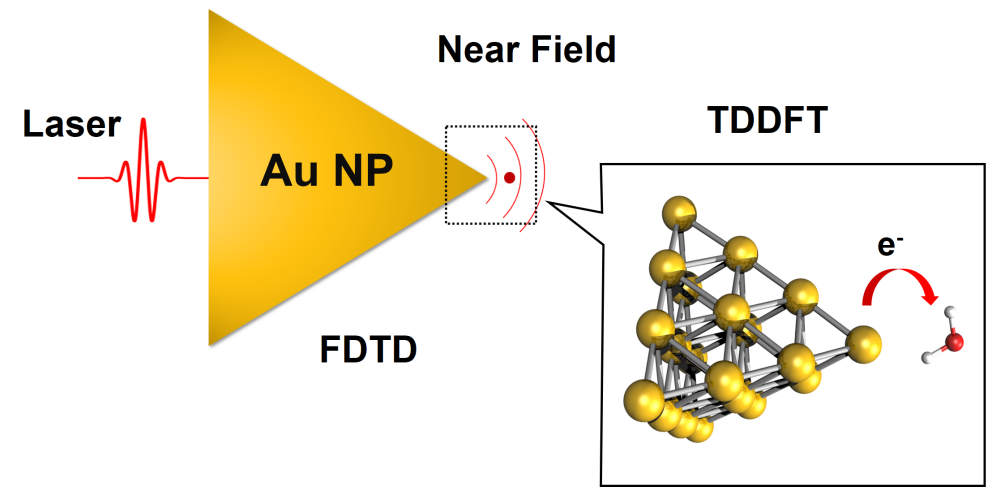

Figure 7: table of contents 